\title{
Miracle Happening In Life: Intra-Abdominal Haemorrhage in Cirrhosis in Pregnancy Managed By Massive Blood Transfusion
}

\author{
Monowara Begum ${ }^{1}$, Housneara Begum², Anisur Rahman ${ }^{3}$
}

\begin{abstract}
Pregnancy is a rare event in patients with cirrhosis. Recent improvements in the treatment of chronic liver disease have resulted in higher conception rates and more successful pregnancy outcomes. Still pregnancy in cirrhosis is associated with high incidence of fetal loss as well as higher maternal mortality both during pregnancy and in the post-partum period. In this paper we described a case of a successful pregnancy in a young woman with advanced cirrhosis facing life threatening condition in her last trimester and perioperative period.
\end{abstract}

\section{Key words}

Pregnancy, cirrhosis, intra-abdominal hemorrhage, massive blood transfusion.

\section{Introduction}

Pregnancy in cirrhotic women is rare. ${ }^{1}$ The exact incidence is unknown but one study revealed that in reproductive age only 45 cases of cirrhosis occurr in every 100,000 women. ${ }^{2}$ But where pregnancy does occur, variceal bleeding due to portal hypertension is a particular risk in the $2^{\text {nd }}$ and $3^{\text {rd }}$ trimester. $^{3}$ Although massive blood transfusion is used for the treatment of uncontrolled hemorrhage, maternal deaths have been reported in advanced cirrhosis mainly due to variceal bleeding. ${ }^{4}$ Spontaneous abortion and increased risk of premature childbirth or stillbirth have been reported in 15-20\% of pregnancies in women with cirrhosis. ${ }^{5}$ We are reporting the case of a successful pregnancy outcome of a woman with decompensated idiopathic cirrhosis complicated with massive intra abdominal bleeding which was managed successfully with whole blood, packed red blood cell, platelet concentrate and fresh frozen plasma in total of 115 units without any transfusion related hazards.

\section{Case}

The patient, a 24 years housewife of middle class family admitted in Apollo Hospitals Dhaka (AHD) as a referred patient at 36 weeks of pregnancy with severe jaundice for safe confinement.
She was mother of one child and had a history of one abortion. She was on regular antenatal check-up out side this hospital. Her pregnancy period was uneventful up to 24 weeks but thereafter she developed jaundice with nausea, vomiting and intermittent fever. As per advice of her obstetrician, she was investigated which showed negative viral markers and was managed accordingly upto 36 weeks of pregnancy. Then she was planned for elective caesarean section outside AHD. But that was postponed due to the apprehension of per operative bleeding hazard and referred to AHD.

On examination she was conscious, oriented, had stable vitals and was severely icteric. Obstetrical examination revealed fundal height 35 weeks, single pregnancy with cephalic presentation with good fetal movement and satisfactory heart rate. From the very beginning she has been managed by a multi-disciplinary team (obstetrician, gastroenterologist, internist, anesthesiologist, hematologists). Thorough investigations revealed deranged LFT (S. bilirubin $6.8 \mathrm{mg} / \mathrm{dl}$, SGPT 48, PT 20.8, albumin $1.7 \mathrm{gm} / \mathrm{dl}$ ) and DIC profile (INR-2.00, APTT-61, D-dimer >4400) but all viral markers were negative. Serum uric acid level was high $(7.5 \mathrm{mg} / \mathrm{dl})$. 
Feto-maternal risk benefit was critically assessed and decision taken for termination of pregnancy keeping arrangement for management of possible complications for surgery and systemic derangement. She delivered a male baby by caesarean section under G/A keeping a drain tube in peritoneal cavity. After 3 hours of post operative period she developed abdominal distension with large hemorrhagic collection in drain tube but there was no PPH. Immediately patient was shifted to surgical ICU. Gradually abdominal distension increased with large amount of drain tube collection. Patient was managed with blood and blood products transfusion. She received 5 units of whole blood, 1 unit PRBC \& 4 units of FFP on that day. USG of whole abdomen was done that revealed large abdomino-pelvic hemorrhagic collection. After that decision was taken for re-exploration by combined team (general surgeon \& obstetrician) on $1^{\text {st }}$ POD. During re-exploration huge amount of clotted \& small amount fresh blood was found in abdominal cavity which was sucked out. Liver bed injury was excluded endoscopically and liver was found to be smaller in size and macro nodular by general surgeon. Uterus was atonic but no bleeding from uterine wound. Anticipating PPH from atonic uterus in already debilitating patient, total abdominal hysterectomy (TAH) was done, abdomen closed with three drain tube kept in situ (one sub-hepatic, one in peritoneal cavity \& another in sub-rectal space). Liver biopsy not attempted considering the emergency situation of the patient. Again patient was shifted to surgical ICU for ventilator support. Following re-exploration patient developed abdominal distension with moderate amount of drain tube collection that was kept in peritoneal cavity. Abdomino aorto angiogram was done to elicit the bleeding point. CT scan of whole abdomen suggested large hematoma within pelvic cavity with upward extension on right side, moderate ascites, and mild fatty infiltration of liver with sludge within gall bladder and features of portal hypertension (mild splenomegaly). Considering patients physical status an emergency medical board was held, then decision was taken for re-exploration of abdomen which was done on 7th POD of TAH by combined team (obstetrician and general surgeon) but no definitive bleeding source was found. During that time four packs were kept in abdominal cavity \& shifted to surgical ICU with ventilator support \& intra-abdominal collection was closely monitored. DT collection was gradually decreasing. Then intra abdominal packs were removed after 3 days of re-exploration. All previous drain tubes were removed. Total 20 units whole blood, 17 units PRBC, 40 units FFP \& 40 units platelet concentrate were transfused. During her ICU period she developed diabetes and wound infection which was treated accordingly. After removal of all stitch she developed burst abdomen which was managed accordingly. Patient was discharged from the hospital after 47 days with stable condition.

\section{Discussion}

Pregnancy is a rare event in patients with cirrhosis. It has traditionally been low for two reasons. First, advanced liver disease does not typically occur until well after most patients have completed their reproductive years, with only 45 cases of cirrhosis occurring in every 100,000 women of reproductive age. Second, Cirrhosis leads to anovulation and amenorrhoea due to many factors that include disturbed oestrogen and endocrine metabolism. ${ }^{6,7}$

When pregnancy is successful in a cirrhotic woman, spontaneous abortion rate, risk of prematurity, and perinatal death rate are all increased. ${ }^{8}$ Review of literature has shown that there is $10-15 \%$ risk of abortion in early pregnancy $^{9,10}$ and 45 out of 69 babies born to cirrhotics survived in the neonatal period. ${ }^{11}$

Cirrhotic patients have a high risk of liver decompensation because of worsening synthetic 
liver function, development of ascites, and hepatic encephalopathy. ${ }^{8}$ Maternal mortality rate as high as $10.5 \%$ has been described in this group. ${ }^{12}$ Maternal prognosis depends on the degree of hepatic dysfunction during pregnancy rather than its cause. ${ }^{13}$ Portal hypertension worsens during pregnancy because of increased blood volume and flow. Portal pressures can also increase because of an increased vascular resistance due to external compression of the inferior vena cava by the gravid uterus. Up to $25 \%$ of patients with varices have a bleeding episode during pregnancy. ${ }^{14}$ The greatest risk is seen in the second trimester, when portal pressure peaks, and during delivery because of the repeated use of the valsalva maneuver to help to expel the fetus. ${ }^{12}$ Rupture of splenic artery aneurysm, although uncommon, could also present in pregnant women with portal hypertension. Hepatic encephalopathy also remains a concern and cause of death in this setting. ${ }^{11}$

In the post-partum period there is high incidence of gastrointestinal hemorrhage responsible for 5 out of 7 deaths during this period. ${ }^{11}$ But, the exception, in our case is there was severe intra abdominal hemorrhage of unknown source which may be due to cirrhosis related low platelet count, DIC related coagulopathy or from portal hypertension related intra abdominal varices which could not be visualized even endoscopically in our settings. Other post-partum complications in cirrhotic patients include uterine hemorrhage, hepatic coma and ascites which were not present in our case. ${ }^{11}$

All cirrhotic patients should undergo variceal screening. Variceal banding before pregnancy, although not proven, is appropriate for high-risk varices. Propranolol has also been used safely in pregnancy but side-effects include fetal growth retardation, neonatal bradycardia, and hypoglycaemia. Terlipressin has not been studied in pregnancy but concerns have been raised about decreased placental perfusion and increased risk of placental abruption. The use of a transjugular intrahepatic portosystemic shunt (TIPS) in extreme cases of variceal bleeding can be considered but has the risk of radiation exposure to the fetus. In a controlled setting vaginal delivery is usually safe and early forceps delivery or vacuum extraction should be considered to prevent any rise in portal pressure due to prolonged straining during labor. ${ }^{15}$ Women with cirrhosis generally tolerate laparotomy poorly; therefore the option for caesarean section should be availed with care and caution. As fetal jeopardy developed we did lower segment caesarean section as an emergency case. An asphyxiated baby boy was delivered by lower segment caesarean section with average birth weight and managed in neonatal intensive care unit. Then our patient had to undergo laparotomy three times for massive internal hemorrhage which was managed with whole blood, packed red blood cell, platelet concentrate and fresh frozen plasma in total of 115 units without any transfusion related hazards.

\section{Conclusion}

Although uncommon, cirrhosis with pregnancy may be encountered in clinical practice. Physicians will need to become aware of the unique risks and complications that they face. These include a potential for life-threatening variceal bleeding, hepatic decompensation, splenic artery aneurysm rupture, postpartum hemorrhage and also intra-abdominal hemorrhage. Medications may need to be adjusted during this time period, as several drugs commonly used for liver disease have potential harmful effects on the fetus or may be transmitted to neonates during breastfeeding.

\section{References}

1. Steven MM, Buckley JD, Mackay IR. Pregnancy in chronic active hepatitis. Q J Med. 1979;48:519-31.

2. Russell MA, Craigo SD. Cirrhosis and portal hypertension in pregnancy. Semin Perinatol. 1998;22:156-165.

3. Bloom S, Webster G. Effects of chronic liver disease on pregnancy. Oxford Hand Book of Gastroenterology and Hepatology. Oxford university press. 2006:77. 
4. Zeeman GG, Moise KJ Jr: Prophylactic banding of severe esophageal varices associated with liver cirrhosis in pregnancy. Obstet Gynecol. 1999;94:842.

5. Lee WM: Pregnancy in patients with chronic liver disease. Gastroenterol Clin North Am. 1992;21:889-903.

6. Russell MA, Craigo SD. Cirrhosis and portal hypertension in pregnancy. Semin Perinatol. 1998;22:156-65.

7. Brunt PW, Kew MC, Scheuer PJ, Sherlock S. Studies in alcoholic liver disease in Britain. I. Clinical and pathological patterns related to natural history. Gut 1974; 15:52-58.

8. Aggarwal N, Sawnhey H, Suril V, Vasishta K, Jha M, Dhiman RK. Pregnancy and cirrhosis of the liver. Aust N Z J Obstet. Gynaecol.1999;39:503-06.
9. Britton RC. Pregnancy and oesophageal varices. American J Sur. 1982;4:421.

10. Varma RR, Michelsohn NH. Pregnancy in cirrhotic and noncirrhotic hypertension. Obstet Gynecol. 1976;50:217.

11. Schreyer P, Caspi E. Cirrhosis, pregnancy and delivery: a review. Obstet Gynecol Survey.1982;37:304.

12. Steven MM. Pregnancy and liver disease. Gut. 1981;22:592-614.

13. Fesenmeier MF, Coppage KH, Lambers DS, Barton JR, Sibai BM. Acute fatty liver of pregnancy in 3 tertiary care centers. Am J Obstet Gynecol. 2005;192:1416-19.

14. Hay JE. Liver disease in pregnancy. Hepatology. 2008;47:1067-76.

15. Cerqui AJ, Haran M, Brodribb R: Implications of liver cirrhosis in pregnancy. Aust $\mathrm{N}$ Z J Obstet Gynaecol. 1998;38:93-5. 